\title{
A Critical Note of Major Parameter Extraction Methods for Proton Exchange Membrane Fuel Cell (PEMFC)
}

\author{
Danyang Li, Bo Yang and Yiming Han* \\ Faculty of Electric Power Engineering, Kunming University of Science and Technology, Kunming, China
}

Keywords: PEMFC, parameter extraction, meta-heuristic algorithm, neural network, zero carbonization

\section{INTRODUCTION}

In the context of the carbon peak and carbon neutral goals, the energy and power industries are undergoing unprecedented changes (Yang et al., 2015). It is particularly noteworthy that carbon neutrality will accelerate the zero-carbonization process of electricity growth, and there is an urgent need to reduce dependence on fossil energy (Yang et al., 2020a). Besides, a proton exchange membrane fuel cell (PEMFC) can convert chemical energy into electrical energy efficiently, without pollution, and is widely used in the fields of mobile equipment such as military, ships, and automotive equipment. However, parameter extraction of the PEMFC is a multivariable, multimode non-linear function optimization problem. Therefore, establishing an accurate and reliable PEMFC model is key to parameter extraction (Zhang et al., 2021). So far, the PEMFC parameter extraction strategy based on meta-heuristic algorithms and artificial neural network (ANN) has attracted widespread attention. However, the practical application of parameter extraction will face many challenges (Huang et al., 2021). Even in the peer-reviewed literature, the parameter extraction strategy is not fully considered, and the potential risks it brings are worth considering. This article clarifies the aforementioned problems and puts forward some opinions on different parameter identification methods. The remaining sections of this article are organized as follows: Proton Exchange Membrane Fuel Cell Modeling indicates parameter extraction using only meta-heuristic algorithms; the parameter extraction based on neural network is investigated in detail in Parameter Extraction Using Only Meta-Heuristic Algorithms; and Parameter Extraction Based on Neural Network presents the discussion and conclusion of this article.

This article was submitted to Process and Energy Systems Engineering,

a section of the journal Frontiers in Energy Research

Received: 14 December 2021 Accepted: 27 December 2021 Published: 02 February 2022

Citation: Li D, Yang B and Han Y (2022) A Critical Note of Major Parameter Extraction Methods for Proton Exchange Membrane Fuel Cell (PEMFC).

Front. Energy Res. 9:835397. doi: 10.3389/fenrg.2021.835397

\section{PROTON EXCHANGE MEMBRANE FUEL CELL MODELING}

As illustrated in Figure 1, the polarization curve characteristics of the PEMFC can help analyze the performance of fuel cells. In addition, the semiempirical model of the PEMFC can describe the working process according to the physical meaning represented by the parameters in the equation, which helps understand and optimize the performance of fuel cells.

In order to accurately analyze the input and output characteristics of the PEMFC, according to the PEMFC electrochemical model, the output voltage can be expressed as follows:

$$
V_{\text {cell }}=E_{\text {nernst }}-V_{\text {act }}-V_{\Omega}-V_{\text {conc }} \text {. }
$$

Moreover, $E_{\text {Nernst }}$ represents the potential obtained by the PEMFC in open thermodynamic equilibrium; it can be described as follows: 


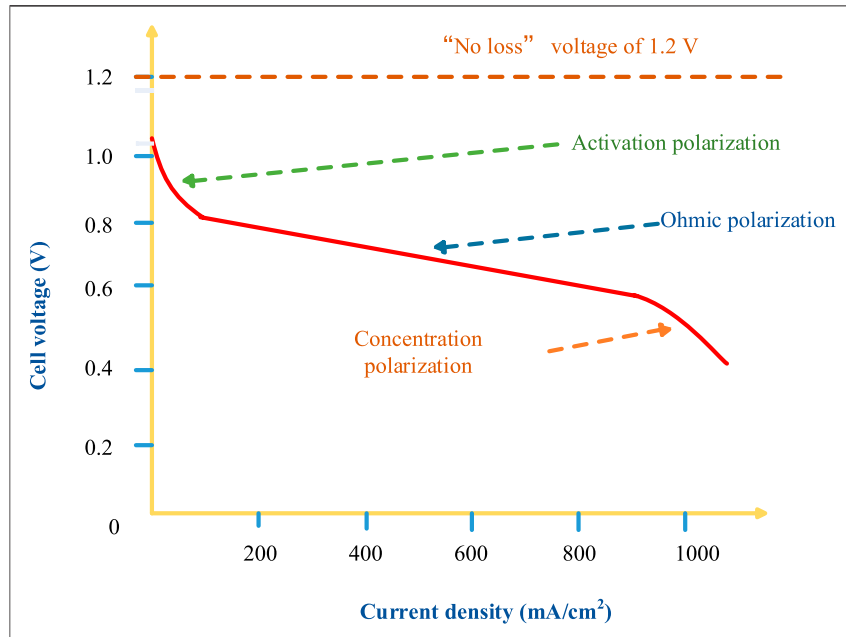

FIGURE 1 | Polarization curve of the PEMFC.

$$
E_{\mathrm{Nernst}}=\frac{\Delta G}{2 F}+\frac{\Delta S}{2 F}\left(T-T_{\mathrm{ref}}\right)+\frac{R T}{2 F}\left[\ln \left(P_{\mathrm{H}_{2}}\right)+\frac{1}{2} \ln \left(P_{\mathrm{O}_{2}}\right)\right] .
$$

$V_{\text {act }}$ denotes the activation overvoltage, and it is determined as follows:

$$
V_{\mathrm{Act}}=\left[\varepsilon_{1}+\varepsilon_{2} T+\varepsilon_{3} T \ln \left(C_{\mathrm{O}_{2}}\right)+\varepsilon_{4} T \ln \left(i_{\mathrm{FC}}\right)\right],
$$

where $\varepsilon_{i}$ denotes the semiempirical coefficients and $i_{\mathrm{FC}}$ is the cathode current.

In addition, the ohmic voltage drop $\left(V_{\Omega}\right)$ is determined as follows:

$$
V_{\Omega}=i_{\mathrm{FC}}\left(R_{\mathrm{m}}+R_{\mathrm{c}}\right)
$$

where $R_{\mathrm{m}}$ indicates the equivalent impedance of a proton membrane and $R_{\mathrm{c}}$ denotes the impedance.

Meanwhile, $V_{\text {conc }}$ indicates the concentration voltage loss. It can be denoted as follows:

$$
V_{\text {conc }}=-b \ln \left(1-\frac{J}{J_{\max }}\right) \text {. }
$$

In summary, it presents seven unknown parameters of the PEMFC, namely, $\varepsilon_{1}, \varepsilon_{2}, \varepsilon_{3}, \varepsilon_{4}, \lambda, R_{\mathrm{c}}$, and $b$. Table 1 shows the lower/upper bound of the unknown parameters for PEMFC models.

Besides, the evaluation standards play an important role in parameter extraction. Therefore, it is necessary to introduce several evaluation standards by appropriately selecting various evaluation standards to correctly verify whether the method can obtain satisfactory results. Among them, root-mean-square error
(RMSE) is very sensitive to the size error of a series of measurements.

Here, RMSE is used as the objective function, which can be written as follows:

$$
\operatorname{RMSE}(z)=\sqrt{\frac{1}{N} \sum_{k=1}^{N}\left(f\left(V_{\mathrm{L}}, I_{\mathrm{L}}, Z\right)\right)^{2}}
$$

where $N$ represents the numbers of $I-V$ data; $I_{\mathrm{L}}$ is defined as output current, and $V_{\mathrm{L}}$ denote output voltage; and $z$ represents the solution vector.

\section{PARAMETER EXTRACTION USING ONLY META-HEURISTIC ALGORITHMS}

As a stochastic method inspired by natural phenomena, metaheuristic algorithms have the characteristics of high flexibility (Yang et al., 2020b), no need to establish precise mathematical models, and can solve the optimization problems of highly nonlinear systems. Thus far, meta-heuristic algorithms have made some progress in improving search capability and efficiency (Yang et al., 2019), which has attracted widespread attention. Since the electrical model of the PEMFC is a complex system with the characteristics of multivariable, strong coupling, and nonlinearity, therefore, the PEMFC parameter extraction strategy based on meta-heuristic algorithms has received extensive attention and has become a very active research field in recent years (Yang et al., 2020c). So far, meta-heuristic algorithms have been roughly divided into four categories, which are based on biology, physics, sociology, and mathematics. In addition, many meta-heuristic algorithms have been applied to PEMFC parameter extraction, for example, antlion optimization algorithm (ALO) (Isa et al., 2019), particle swarm optimization algorithm (PSO) (Ye et al., 2009), biogeographybased optimization (BBO) (Gong and Cai, 2014), improved beetle antennae search (IBAS) (Sun et al., 2020), hybrid artificial bee colony algorithm (ABC) (Oliva et al., 2014), vortex search algorithm (VSA) (Fathy et al., 2020), differential evolution (DE) (Chakraborty et al., 2012), month flame optimizer algorithm (MFO) (Messaoud et al., 2020), multi-verse optimizer (MVO) (Zhao et al., 2016), gray wolf optimizer (GWO) (Yang et al., 2017), genetic algorithm (GA) (Ohenoja and Leiviskä, 2010), flower pollination algorithm (FPA) (Priya and Rajasekar, 2019), and equilibrium optimizer (EO) (Seleem

\begin{tabular}{|c|c|c|c|c|c|c|c|}
\hline Model parameter & $\varepsilon_{1}$ & $\varepsilon_{2}$ & $\varepsilon_{3}$ & $\varepsilon_{4}$ & $\lambda$ & $\mathbf{R}_{\mathrm{c}}$ & b \\
\hline Lower bound $X_{I}$ & -1.1997 & 0.001 & $3.6 \times 10^{-5}$ & -0.00026 & 10 & 0.0001 & 0.0136 \\
\hline Upper bound $X_{\mathrm{u}}$ & -0.8531 & 0.005 & $9.8 \times 10^{-5}$ & -0.0000954 & 23 & 0.0008 & 0.5 \\
\hline
\end{tabular}
et al., 2021).

In addition, it should be noted that meta-heuristic algorithms still have some shortcomings in terms of convergence speed and computational efficiency, and it is easy to fall into the local 
optimum during the optimization process (Zhang et al., 2016). Thus, in order to further improve the performance of the parameter extraction of meta-heuristic algorithms, a series of improved and mixed versions are proposed to improve the search efficiency and robustness and avoid falling into local optimization. In Niu et al. (2014); Zhang et al. (2015), the migration operator can effectively improve global search efficiency but lacks local deeply digging capabilities, which can easily lead to premature convergence. Therefore, combining the mutation theory and chaos strategy of the differential evolution (DE) algorithm with the original mutation strategy of the biogeography-based optimization (BBO) algorithm, the $\mathrm{BBO}$ with mutation strategy algorithm (BBO-M) was proposed, which effectively improves the global search efficiency, enhances the convergence speed, and provides a new research idea for PEMFC parameter extraction. Zhang and Wang (2013); Niu et al. (2014); Liu et al. (2020) developed an improved genetic algorithm (GA) based on adaptive RNA, called adaptive RNA (ARNA-GA), which uses an adaptive strategy to crossover and mutate according to the differences between different individuals. Therefore, compared with GA, ARNA-GA avoids premature convergence and improves the efficiency of global search, which is worthy of reference. In addition, in order to improve computational efficiency and global search capabilities, Yao et al. (2015; Chen et al. (2018; Fathy et al. (2020) studied a hybrid vortex search differential evolution (VSDE) algorithm, in which the control parameters are jointly adjusted by the DE and the vortex search algorithm (VSA). This makes VSDE highly reliable and effective in PEMFC parameter extraction. Specifically, the principle of the JAYA algorithm to improve the convergence speed is iterated at the same time until the optimal solution is achieved, avoiding low-quality solutions, and only needs to specify the two parameters of population size and algebra. At the same time, considering that the Nelder-Mead (NM) simplex strategy has the characteristics of simple structure and strong local development capabilities, Yu et al. (2019); Zhou et al. (2020) proposed a simple two-stage eagle strategy based on the JAYA algorithm and the Nelder-Mead simplex algorithm (JAYA-NM). The results show that the JAYA-NM algorithm exhibits satisfactory convergence speed and accuracy in PEMFC parameter extraction.

However, due to the inherent defect of randomness in metaheuristic algorithms, the quality of optimal solution varies with the number of iterations and the number of populations (Xiong et al., 2021). The weight parameters assigned to algorithms should also be carefully chosen. For different experimental environments, it is necessary to set the algorithm parameters in a targeted manner in order to weigh the calculation amount of the algorithm and the quality of the solution. In addition, all the aforementioned methods can only be used for parameter extraction when the experimental data are sufficient and the influence of experimental data noise is not considered, which limits the accurate extraction of PEMFC parameters and cannot be accurately modeled. Therefore, it is a new and challenging task to extract PEMFC parameters under insufficient experimental data and noisy experiment environments.

\section{PARAMETER EXTRACTION BASED ON NEURAL NETWORK}

Parameter extraction methods using neural network are mainly aimed to make use of artificial neural network and its derivatives to improve the performance of PEMFC parameter extraction (Kalyan and Rao, 2021). The following outstanding advantages of ANN have attracted great attention in recent years: 1) It can be perfectly close to a complex non-linear relationship; 2) all qualitative information is stored in each neuron in the network, which has strong robustness and fault tolerance; 3) parallel distributed processing methods can quickly perform many operations; 4) can learn and adapt to uncertain systems; and 5) can handle both quantitative and qualitative knowledge.

At present, there are many kinds of neural network research methods, and the most fruitful research includes the BP algorithm of multilayer network, Hopfield network model, adaptive resonance theory, and self-organizing feature mapping theory. Besides, the accuracy of PEMFC parameter extraction results depends on the accuracy and reliability of raw data (Liu et al., 2021). However, in the procession of PEMFC parameter extraction via meta-heuristic algorithms, some data problems affecting the accuracy of parameter extraction are ineluctable, for example, the necessary raw data are not so enough that the reliability of the extracted parameters is reduced. In addition, the matter of noises in the original voltage and current data is also a common and inevitable problem. Thus, the introduction of neural network and its derivatives into the parameter extraction method can not only make the parameter extraction more adaptive for the raw data but also provide a more reliable fitness function for parameter extraction.

Thus far, Bayesian regularization neural network (BRNN) (Yang et al., 2021a), extreme learning machine (ELM) (Yang et al., 2021b), Levenberg-Marquardt backpropagation (LMBP) algorithm (Yang et al., 2021c), Elman neural network (ENN), deep belief network (DBN), support vector machine (SVM), random forest (RF), feedforward backpropagation (FFBP) (Wilberforce and Olabi, 2020), hidden semi-Mark model (HSMM) (Wu et al., 2017), and several other methods based on the neural network and its derivatives are applied to the parameter extraction research of fuel cells. Yang et al. (2021a) provide a novel idea for the research on parameter extraction of the PEMFC with noisy data, in which, due to the influence of data noise on the accuracy of extracted parameters, BRNN-based meta-heuristic algorithms are proposed to filter out the noises and prevent the "overfitting" phenomenon, improving the performance of PEMFC parameter extraction. Literature Yang et al. (2021b); Yang et al. (2021c) combines ELM and LMBP with several prominent meta-heuristic algorithms. Due to insufficient voltage and current data provided by the manufacturer, the accuracy of parameter extraction will be reduced. Among them, ELM training data compensates for the lack of data in parameter extraction and improves the accuracy of parameter extraction, but there is no standard for the number of original voltage and current data, that is, how much data is needed to meet the requirements under a certain application background 
application requirement. In work Wilberforce and Olabi (2020) used artificial neural networks to compare the FFBP and data processing grouping method $(\mathrm{GMDH})$ to determine voltage and current. The research result shows that GMDH neural network is better than FFBP neural network. Wu et al. (2017) combine an HSMM and empirical model, proposing an improved prediction model to predict the remaining service life of fuel cells. The experimental results show that compared with the existing fuel cell prediction methods, the prediction model has higher prediction accuracy and faster prediction speed. In addition, it is noticeable that these strategies are all dedicated to improving the accuracy (Erdiwansyah et al., 2021; Padhy and Panda, 2021; Yang et al., 2021d), stability, and efficiency of PEMFC parameter extraction in various adverse conditions but not further delving into the degree of influence of these adverse effects on the parameter extraction results and to what extent the affected results can be regarded as acceptable results (Petrone et al., 2013; Chatrattanawet et al., 2017; Muniappan, 2021).

However, the literature did not figure out the specific impact of noise data on PEMFC parameter extraction and the impact of environmental factors on the anti-interference ability of parameter extraction (Chen et al., 2020; Guo et al., 2020). For example, the noise range that the data can withstand in order to ensure the accuracy of parameter extraction in a certain application environment should be determined. In addition, there is an urgent need to develop a metaheuristic algorithm based on neural network, which mainly aimed to make use of an artificial neural network and its derivatives to improve the PEMFC parameter extraction accuracy.

\section{REFERENCES}

Chakraborty, U. K., Abbott, T. E., and Das, S. K. (2012). PEM Fuel Cell Modeling Using Differential Evolution. Energy 40, 387-399. doi:10.1016/ j.energy.2012.01.039

Chatrattanawet, N., Hakhen, T., Kheawhom, S., and Arpornwichanop, A. (2017). Control Structure Design and Robust Model Predictive Control for Controlling a Proton Exchange Membrane Fuel Cell. J. Clean. Prod. 148, 934-947. doi:10.1016/j.jclepro.2017.02.033

Chen, J., Yao, W., Zhang, C., Ren, Y., and Jiang, L. (2018). Design of Robust MPPT Controller for Grid-Connected PMSG-Based Wind Turbine via Perturbation Observation Based Nonlinear Adaptive Control. Renew. Energ. 101, 34-51. doi:10.1016/j.renene.2018.11.048

Chen, K., Laghrouche, S., and Djerdir, A. (2020). Proton Exchange Membrane Fuel Cell Prognostics Using Genetic Algorithm and Extreme Learning Machine. Fuel Cells 20 (3), 263-271. doi:10.1002/fuce.201900085

Erdiwansyah, E., Mahidin, A. T., Husin, H., Nasaruddin, S., Zaki, M., and Muhibbuddin (2021). A Critical Review of the Integration of Renewable Energy Sources with Various Technologies. Prot. Control. Mod. Power Syst. 6, 1-18. doi:10.1186/s41601-021-00181-3

Fathy, A., Elaziz, M. A., and Alharbi, A. G. (2020). A Novel Approach Based on Hybrid Vortex Search Algorithm and Differential Evolution for Identifying the Optimal Parameters of PEM Fuel Cell. Renew. Energ. 146, 1833-1845. doi:10.1016/j.renene.2019.08.046

Gong, W., and Cai, Z. (2014). Parameter Optimization of PEMFC Model with Improved Multi-Strategy Adaptive Differential Evolution. Eng. Appl. Artif. Intellig. 27, 28-40. doi:10.1016/j.engappai.2013.07.016

Guo, H. B., Tao, H., Salih, S. Q., and Zaher, M. Y. (2020). Optimized Parameter Estimation of a PEMFC Model Based on Improved Grass Fibrous Root

\section{DISCUSSION AND CONCLUSION}

A reliable parameter extraction strategy is particularly important for PEMFC system performance evaluation and optimization, but it is still in the research and development stage. The efficiency and engineering practicability of this technology are the main challenges and are listed as follows:

Insufficient training data and excessive noise may lead to overfitting of the parameter extraction results, and operating cycles, thinning of the catalyst, and poisoning will lead to shortened battery life and performance degradation. Therefore, it can provide an effective and efficient tool to solve these problems and can be applied to life prediction and fault diagnosis based on the accurate extraction of experimental parameters. At the same time, it is necessary to develop and apply some new hybrid methods by combining the advantages of different meta-heuristic algorithms and neural networks to further obtain better performance. The hybrid method is a promising optimization method, which provides a new method to improve PEMFC parameter extraction accuracy. In addition, the proposed method is only evaluated under simulation conditions. Therefore, the next step for the researcher is to study its application to actual experimental data to test its actual performance.

\section{AUTHOR CONTRIBUTIONS}

DL helped with writing the original draft and editing. BY contributed to conceptualization. YH assisted with visualization and contributed to the discussion of the topic.

Optimization Algorithm. Energ. Rep. 6, 1510-1519. doi:10.1016/ j.egyr.2020.06.001

Huang, S., Wu, Q., Liao, W., Wu, G., Li, X., and Wei, J. (2021). Adaptive DroopBased Hierarchical Optimal Voltage Control Scheme for vsc-hvdc Connected Offshore Wind Farm. IEEE Trans. Ind. Inf. 17 (12), 8165-8176. doi:10.1109/ tii.2021.3065375

Isa, Z. M., Nayan, N. M., Arshad, M. H., and Kajaan, N. A. M. (2019). Optimizing Pemfc Model Parameters Using Ant Lion Optimizer and Dragonfly Algorithm: A Comparative Study. Int. J. Electr. Comput. Eng. 9 (6), 5295. doi:10.11591/ ijece.v9i6.pp5295-5303

Kalyan, C. H. N. S., and Rao, G. S. (2021). Impact of Communication Time Delays on Combined LFC and AVR of a Multi-Area Hybrid System with IPFC-RFBs Coordinated Control Strategy. Prot. Control. Mod. Power Syst. 6, 7. doi:10.1186/ s41601-021-00185-Z

Liu, J., Yao, W., Wen, J., Fang, J., Jiang, L., He, H., et al. (2020). Impact of Power Grid Strength and PLL Parameters on Stability of Grid-Connected DFIG Wind Farm. IEEE Trans. Sustain. Energ. 11 (1), 545-557. doi:10.1109/ tste.2019.2897596

Liu, S., Zhou, C., and Guo, H. (2021). Operational Optimization of a BuildingLevel Integrated Energy System Considering Additional Potential Benefits of Energy Storage. Prot. Control. Mod. Power Syst. 6, 4. doi:10.1186/s41601-02100184-0

Messaoud, R. B., Midouni, A., and Hajji, S. (2020). PEM Fuel Cell Model Parameters Extraction Based on Moth-Flame Optimization. Chem. Eng. Sci. 229, 81-89. doi:10.1016/j.ces.2020.116100

Muniappan, M. (2021). A Comprehensive Review of DC Fault protection Methods in HVDC Transmission Systems. Prot. Control. Mod. Power Syst. 6, 1. doi:10.1186/s41601-020-00173-9

Niu, Q., Zhang, L., and Li, K. (2014). A Biogeography-Based Optimization Algorithm with Mutation Strategies for Model Parameter Estimation of 
Solar and Fuel Cells. Energ. Convers. Manag. 86 (1), 1173-1185. doi:10.1016/ j.enconman.2014.06.026

Ohenoja, M., and Leiviskä, K. (2010). Validation of Genetic Algorithm Results in a Fuel Cell Model. Int. J. Hydrog. Energy 35, 12618-12625. doi:10.1016/ j.ijhydene.2010.07.129

Oliva, D., Cuevas, E., and Pajares, G. (2014). Parameter Identification of Solar Cells Using Artificial Bee colony Optimization. Energy 72, 93-102. doi:10.1016/ j.energy.2014.05.011

Padhy, S., and Panda, S. (2021). Application of a Simplified Grey Wolf Optimization Technique for Adaptive Fuzzy PID Controller Design for Frequency Regulation of a Distributed Power Generation System. Prot. Control. Mod. Power Syst. 6, 2. doi:10.1186/s41601-021-00180-4

Petrone, R., Zheng, Z., Hissel, D., Pera, M. C., Pianese, C., Sorrentino, M., et al. (2013). A Review on Model-Based Diagnosis Methodologies for PEMFCs. Int. J. Hydrog. Energy 38 (17), 7077-7091. doi:10.1016/j.ijhydene.2013.03.106

Priya, K., and Rajasekar, N. (2019). Application of Flower Pollination Algorithm for Enhanced Proton Exchange Membrane Fuel Cell Modelling. Int. J. Hydrog. Energy 44 (33), 18438-18449. doi:10.1016/j.ijhydene.2019.05.022

Seleem, S. I., Hasanien, H. M., and El-Fergany, A. A. (2021). Equilibrium Optimizer for Parameter Extraction of a Fuel Cell Dynamic Model. Renew. Energ. 169, 117-128. doi:10.1016/j.renene.2020.12.131

Sun, K., Yao, W., Fang, J., Ai, X., Wen, J., and Cheng, S. (2020). Impedance Modeling and Stability Analysis of Grid-Connected DFIG-Based Wind Farm with a VSC-HVDC. IEEE J. Emerg. Sel. Top. Power Electron. 8 (2), 1375-1390. doi:10.1109/jestpe.2019.2901747

Wilberforce, T., and Olabi, A. G. (2020). Proton Exchange Membrane Fuel Cell Performance Prediction Using Artificial Neural Network. Int. J. Hydrogen Energ. 46 (8), 6037-6050. doi:10.1016/j.ijhydene.2020.07.263

Wu, X. J., Ye, Q. W., and Wang, J. H. (2017). A Hybrid Prognostic Model Applied to SOFC Prognostics. Int. J. Hydrog. Energy 42 (39), 25008-25020. doi:10.1016/ j.ijhydene.2017.08.114

Xiong, Y., Yao, W., Wen, J., Lin, S., Ai, X., Fang, J., et al. (2021). Two-Level Combined Control Scheme of VSC-MTDC Integrated Offshore Wind Farms for Onshore System Frequency Support. IEEE Trans. Power Syst. 36 (1), 781-792. doi:10.1109/tpwrs.2020.2998579

Yang, B., Jiang, L., Yao, W., and Wu, Q. H. (2015). Perturbation Estimation Based Coordinated Adaptive Passive Control for Multimachine Power Systems. Control. Eng. Pract. 44, 172-192. doi:10.1016/j.conengprac.2015.07.012

Yang, B., Zhang, X., Yu, T., Shu, H., and Fang, Z. (2017). Grouped Grey Wolf Optimizer for Maximum Power point Tracking of Doubly-Fed Induction Generator Based Wind Turbine. Energ. Convers. Manag. 133, 427-443. doi:10.1016/j.enconman.2016.10.062

Yang, B., Zhong, L., Zhang, X., Shu, H., Yu, T., Li, H., et al. (2019). Novel Bio-Inspired Memetic Salp Swarm Algorithm and Application to MPPT for PV Systems Considering Partial Shading Condition. J. Clean. Prod. 215, 1203-1222. doi:10.1016/j.jclepro.2019.01.150

Yang, B., Wang, J., Zhang, X., Wang, J., Shu, H., Li, S., et al. (2020). Applications of Battery/Supercapacitor Hybrid Energy Storage Systems for Electric Vehicles Using Perturbation Observer Based Robust Control. J. Power Sourc. 448, 227444. doi:10.1016/j.jpowsour.2019.227444

Yang, B., Wang, J., Zhang, X., Yu, T., Yao, W., Shu, H., et al. (2020). Comprehensive Overview of Meta-Heuristic Algorithm Applications on PV Cell Parameter Identification. Energ. Convers. Manag. 208, 112595. doi:10.1016/ j.enconman.2020.112595

Yang, B., Zhu, T., Wang, J., Shu, H., Yu, T., Zhang, X., et al. (2020). Comprehensive Overview of Maximum Power Point Tracking Algorithms of PV Systems under Partial Shading Condition. J. Clean. Prod. 268, 121983. doi:10.1016/ j.jclepro.2020.121983

Yang, B., Li, D. Y., Zeng, C. Y., Chen, Y. J., Guo, Z. X., Wang, J. B., et al. (2021). Parameter Extraction of PEMFC via Bayesian Regularization Neural Network Based Meta-Heuristic Algorithms. Energy 228, 120592. doi:10.1016/ j.energy.2021.120592
Yang, B., Chen, Y. J., Guo, Z. X., Wang, J. B., Zeng, C. Y., Li, D. Y., et al. (2021) Levenberg-Marquardt Backpropagation Algorithm for Parameter Identification of Solid Oxide Fuel Cells. Int. J. Energ. Res. 45, 17903-17923. doi:10.1002/er.6929

Yang, B., Guo, Z. X., Chen, Y. J., Wang, J. B., Wang, J. T., Zeng, C. Y., et al. (2021). Extreme Learning Machine Based Meta-Heuristic Algorithms for Parameter Extraction of Solid Oxide Fuel Cells. Appl. Energ. 303, 117630. doi:10.1016/ j.apenergy.2021.117630

Yang, B., Zeng, C. Y., Li, D. Y., Chen, Y. J., Guo, Z. X., Wang, J. B., et al. (2021). Parameter Identification of Proton Exchange Membrane Fuel Cell via Levenberg-Marquardt Backpropagation Algorithm. Int. J. Hydrog. Energy 44 (28), 22998-23012. doi:10.1016/j.ijhydene.2021.04.130

Yao, W., Jiang, L., Wen, J., Wu, Q., and Cheng, S. (2015). Wide-Area Damping Controller for Power System Interarea Oscillations: A Networked Predictive Control Approach. IEEE Trans. Control. Syst. Tech. 23 (1), 27-36. doi:10.1109/ tcst.2014.2311852

Ye, M., Wang, X., and Xu, Y. (2009). Parameter Identification for Proton Exchange Membrane Fuel Cell Model Using Particle Swarm Optimization. Int. J. Hydrog. Energy 34, 981-989. doi:10.1016/j.ijhydene.2008.11.026

Yu, K. J., Qu, B. Y., Yue, C. T., Ge, S. L., Chen, X., and Liang, J. (2019). A Performance-Guided JAYA Algorithm for Parameters Identification of Photovoltaic Cell and Module. Appl. Energy 237, 241-257. doi:10.1016/ j.apenergy.2019.01.008

Zhang, L., and Wang, N. (2013). An Adaptive RNA Genetic Algorithm for Modeling of Proton Exchange Membrane Fuel Cells. Int. J. Hydrog. Energ. 38 (1), 219-228. doi:10.1016/j.ijhydene.2012.10.026

Zhang, X., Yu, T., Yang, B., Zheng, L., and Huang, L. (2015). Approximate Ideal Multi-Objective Solution $\mathrm{Q}(\lambda)$ Learning for Optimal Carbon-Energy Combined-Flow in Multi-Energy Power Systems. Energ. Convers. Manag. 106, 543-556. doi:10.1016/j.enconman.2015.09.049

Zhang, X., Yu, T., Yang, B., and Li, L. (2016). Virtual Generation Tribe Based Robust Collaborative Consensus Algorithm for Dynamic Generation Command Dispatch Optimization of Smart Grid. Energy 101, 34-51. doi:10.1016/j.energy.2016.02.009

Zhang, K., Zhou, B., Or, S. W., Li, C., Chung, C. Y., and Voropai, N. I. (2021). Optimal Coordinated Control of Multi-Renewable-to-Hydrogen Production System for Hydrogen Fueling Stations. IEEE Trans. Ind. Appl. 1. doi:10.1109/ TIA.2021.3093841

Zhao, D., Dou, M., Zhou, D., and Gao, F. (2016). Study of the Modeling Parameter Effects on the Polarization Characteristics of the PEM Fuel Cell. Int. J. Hydrog. Energy 41 (47), 22316-22327. doi:10.1016/j.ijhydene.2016.09.112

Zhou, B., Fang, J., Ai, X., Yang, C., Yao, W., and Wen, J. (2020). Dynamic Var Reserve-Constrained Coordinated Scheduling of LCC-HVDC Receiving-End System Considering Contingencies and Wind Uncertainties. IEEE Trans. Sustain. Energ. 12 (1), 469-481. doi:10.1109/TSTE.2020.3006984

Conflict of Interest: The authors declare that the research was conducted in the absence of any commercial or financial relationships that could be construed as a potential conflict of interest.

Publisher's Note: All claims expressed in this article are solely those of the authors and do not necessarily represent those of their affiliated organizations, or those of the publisher, the editors, and the reviewers. Any product that may be evaluated in this article, or claim that may be made by its manufacturer, is not guaranteed or endorsed by the publisher.

Copyright (C) $2022 \mathrm{Li}$, Yang and Han. This is an open-access article distributed under the terms of the Creative Commons Attribution License (CC BY). The use, distribution or reproduction in other forums is permitted, provided the original author(s) and the copyright owner(s) are credited and that the original publication in this journal is cited, in accordance with accepted academic practice. No use, distribution or reproduction is permitted which does not comply with these terms. 